\title{
La medición de la autoestima en adolescentes y adultos: una propuesta desde el modelo de Harris Clemens
}

Dr. Orlando Cerna Dorregaray

\section{Introducción}

Si bien la investigación sobre la medición de la autoestima es amplia y es una variable que se relaciona con otras como pueden ser, la agresividad, el rendimiento académico, el éxito o fracaso escolar; hemos podido constatar que los fundamentos teóricos y los instrumentos utilizados en la mayoría de los estudios son mayormente los mismos. Así tenemos las investigaciones hechas con niños y adolescentes en los últimos diez años, han partido de las propuestas teóricas de Branden, Milicic, Misitu, y utilizando escalas como la de Rosemberg y Coopersmith. Dentro de los estudios que realizamos tuvimos la oportunidad de conocer una propuesta teórica importante y diferente, que ha sido útil en la explicación de la autoestima en niños y su relación por ejemplo con el éxito escolar. Esta es la propuesta de Harris Clemens (1998) sobre la autoestima. Clemens sostiene que la autoestima es la satisfacción que sentimos de nosotros mismos cuando hemos podido satisfacer cuatro necesidades básicas: la vinculación, la singularidad, el poder y las pautas. Este modelo, que nos parece completo, ayuda no sólo a entender cómo se estructura la autoestima sino que nos permite establecer acciones para desarrollarla, al lograr la satisfacción o el desarrollo pleno de estos cuatro componentes. Fue así que nos preguntamos si este modelo, aplicado más a nivel de niños, puede también ser aplicado para entender los problemas que se dan a nivel de la autoestima de adolescentes y adultos. Partiendo entonces siempre de esta perspectiva teórica, y de esta necesidad decidimos elaborar una escala para medir la autoestima. La misma nos ayudaría a describir la autoestima de adolescentes y adultos y al mismo tiempo ofrecer una escala que luego de someterse a los rigurosos análisis de validez y confiabilidad pueda ser útil para medir la autoestima de adolescentes y adultos que día a día se enfrentan a dificultades en sus relaciones interpersonales por no haber logrado desarrollar una autoestima alta. Además a partir de la medición de la autoestima se pueden establecer planes de mejora que los conduzcan al logro de una autoestima que los aleje de la problemática señalada. Destacamos en la elaboración de la escala la participación de los estudiantes de internado de la primera y segunda promoción 
de la carrera de psicología de nuestra universidad. Ellos participaron en la construcción de la escala como parte de su curso de internado. Por ello, la hemos denominado Escala de Autoestima 1-2 PSIUMCH y la presentamos hoy a la comunidad universitaria como una propuesta para ser sometida al rigor científico de la validez y confiabilidad que todo instrumento psicológico debe tener. Es por lo tanto una propuesta de medición con un marco teórico de referencia y que estamos seguros se convertirá en un instrumento de ayuda a los psicólogos que reconocen en la autoestima a uno de los factores más importantes y nucleares en la personalidad de todos los individuos.

\section{Antecedentes teóricos sobre la autoestima y su medición}

Uno de los estudios más conocidos sobre autoestima es el de Branden (1995) que relaciona la autoestima con la capacidad de sentirse capaz de realizar las cosas, lo que en términos de Clemens (1995) hace referencia al poder, como aquellas habilidades y competencias que tiene el individuo y que lo hacen sentirse contento de poseerlas y de todo aquello que puede lograr con dichas habilidades. Otro aspecto que señala Branden es el de la autodignidad, que se refiere al sentirse seguro que tiene derecho a ser feliz y que puede expresar sus sentimientos de alegría por ejemplo.

Por otro lado, Clark, Clemens y Bean (1999) cuando se refieren a la autoestima en los adolescentes, señalan que esta es expresión de tres componentes importantes que son la imagen corporal, donde el adolescente sabe cuidar su cuerpo; la autovaloración, que implica el considerarse valioso pero sabiendo que hay limitaciones que nos deben llevar a crecer y mejorar; y la capacidad y habilidades, como la confianza en la capacidad intelectual, en su conducta y sentimientos relacionados a su existencia.

Otro investigador teórico clásico sobre la autoestima es Bernard (2004) quien señala que la autoestima es una autoaprobación afectiva de uno mismo que debe ser coherente con lo que realmente somos, es en sí una autopercepción.

Los estudios de Castañeda (2013) indican, citando a Campbell, Chew y Scratchley (1991) que la autoestima es un elemento del sí mismo que se relaciona con la dimensión evaluativa. Es decir la autoestima es una actitud que se produce cuando el sí mismo se ve evaluado por los demás. Por otro lado, Castañeda citando a Kihlstrom y Kantor, 1983, Markus, 1977,1980; en Campbell, 1991, encuentran que existe una relación entre el bienestar psicológico del sí mismo y la autoestima.

Este estudio de Castañeda también señala, citando a Ivannova (2007) que existe una relación entre la autoestima y los problemas de internalización. Así mismo Castañeda también cita a Chen, Hong y Yang (2010) quienes encuentran una relación entre la baja autoestima y las ideas de suicidio que están relacionadas a situaciones de la vida que pueden contribuir a formular o en pensar en suicidarse. Otros autores citados por Castañeda, son Orth y Robins (2008) quienes descubren relaciones entre la baja autoestima y depresión en adolescentes y adultos.

Castañeda cita además a Gómez-Bustamante y Cogollo (2010) para indicar que se han encontrado relación entre alta religiosidad, autoestima y familias funcionales y el bienestar en general de los adolescentes, lo cual confirma la importancia de la autoestima alta en la adolescencia como un predictor que los aleja en el futuro de la psicopatología. Hemos podido constatar lo importante de 
los estudios de Castañeda en la medida que se revaloriza la autoestima cuando se encuentran en las investigaciones las relaciones con otras variables tan vitales para toda persona.

Para Robles (2012) citando a Calero (2000) y a López (2009) sostiene que la autoestima es una parte de la personalidad, que es fundamental para superar los obstáculos que se le presentan a un individuo en la vida y que están relacionados con la responsabilidad, el aprendizaje y la confianza en uno mismo.

Por otro lado, Robles citando a Oñate y García (2007) sostiene que la autoestima es una fuerza intrínseca que lleva al ser humano a la búsqueda del concepto de sí mismo y a su autoafirmación como un ser de valía ante el mundo.

Rodriguez y Caño (2012) realizan un estudio sobre el autoconcepto, la autoestima y la percepción de autovalia donde encuentran que son tres conceptos fuertemente relacionados entre sí. En primer lugar tenemos el autoconcepto que se basa en las creencias sobre uno mismo. Luego se encuentra la autoestima que se relaciona con lo valorativo y afectivo según los estudios de Rodriguez y Caño citando a Marsh y Craven (2006). Estos autores encuentran que la autoestima es cambiante de acuerdo a los acontecimientos que ocurren a lo largo de la vida del individuo y en el caso de los adolescentes se ve reflejada en el refuerzo que hacen de su autoestima cuando las circunstancias y las situaciones en las que actúan los hacen sentirse valiosos para los demás. Esto último tiene que ver con lo que se ha denominado como percepción de la valía y que se encuentra fluctuando en la adolescencia. Estos, los adolescentes, se quedan fijados a estos momentos positivos en su vida y se constituyen por lo tanto en parte de su autoestima.

Otros hallazgos interesantes sobre la autoestima que son señalados en el estudio de Rodriguez y Caño se refieren a la relación de la autoestima con algunos síntomas psicopatólogicos en jóvenes universitarios. Asimismo estos autores encuentran citando a Garaigordobil (2008); García, Zaldivar, Lopez y Molina (2009); Kavas (2009) y Robins (2010) relación entre la baja autoestima y la procrastinación por un lado y por otro el incremento del consumo de alcohol en poblaciones de jóvenes universitarios. Hallazgo que también nos debe llamar la atención para prevenir estas dificultades en la población universitaria.

En este mismo estudio Rodriguez y Caño citan a Trzesniewski (2006) y sostienen que la importancia de una autoestima saludable es nuclear en la adolescencia para prevenir en la vida adulta problemas relacionados con la salud física y mental. Se encontraron dificultades a nivel laboral y económico, así como situaciones que tienen que ver con conductas antisociales en estos adultos que a través del estudio longitudinal de Trzesniewski fueron evaluados en su adolescencia con una autoestima baja en comparación con el grupo que obtuvo en la adolescencia una alta autoestima. Este hallazgo predictor nos debería preocupar para trabajar a nivel del refuerzo de una autoestima saludable en jóvenes universitarios.

Muñoz (2011) citando a lannizzotto (2009) define a la autoestima como el sentimiento que se origina en nosotros luego de darnos cuenta de la persona que somos. Para este autor la autoestima es la unión de conocimiento y afectos. Por un lado tenemos el componente del autoconcepto cuando hablamos de conocimiento de sí mismo y por el otro al lado afectivo que es consecuencia 
de la estima o del rechazo de los demás. Esto explica el autor es la base para otros sentimientos y aquí se puede explicar cómo existan personas que aparentemente tienen muchas personas que las rodean y las quieran o estén llenas de bienes materiales y de comodidad que se sientan desestimadas, esto existe porque en la base de sus afectos hay una dificultad o un déficit en su autoestima.

Todos los estudios previos hasta aquí presentados, demuestran la importancia de la autoestima en la vida de todos. Sin embargo, si se han demostrado la relación de la autoestima con todas las variables planteadas, cuestionamos porque seguimos con las dificultades a nivel de la autoestima. Creemos que solo la hemos enfocado desde lo aprendido, desde lo cognitivo, enfoques que no están equivocados, sin embargo faltaría una propuesta que se sume a estos enfoques, o que la complemente, que veamos una propuesta integral. Es aquí donde la propuesta teórica de Clemens (1999), que parte de las necesidades, de la satisfacción de las mismas y desde ella podemos ver la autoestima de manera más completa y sobre todo más vista desde el desarrollo. Esta propuesta la desarrollaremos a mayor profundidad en un apartado posterior.

\section{Sobre la medición de la autoestima}

Muñoz (2011) indica que la mayoría de las pruebas utilizadas para medir la autoestima son autoreportes y que las tendencias de los mismos llevan a ubicarse a los que contestan los mismos en niveles medios o altos de autoestima, lo cual parece corroborar la idea que somos muy condescendientes con nosotros mismos al autoevaluarnos y esto nos lleva a vernos mejor de lo que realmente somos o nos sentimos.

Muñoz citando a los estudios realizados por Trzesniewski (2003) y que confirman la revisión que hemos realizado en este estudio, señalan que los instrumentos más utilizadas se encuentran la Escala de Autoestima de Rosenberg y el Inventario de Autoestima de Coopersmith. Luego vienen la Subescala de Autovalía Global del Perfil de Autopercepción para niños de Harter. Finalmente se encuentra el Cuestionario de Autodescripción de Marsh.

En el Perú se ha utilizado mucho la Escala de Auotestima de Coopersmith, que fue traducida y validada por primera vez por Panizo (1985), según el propio Coopersmith (1967) la autoestima se refiere a la evaluación que nos hacemos de nosotros mismos y el nivel de valor que tenemos, esto pasa por aspectos relacionados con sentirse capaz, importante y valioso para los demás.

En el caso de Rosemberg, su escala también ha sido utilizada en nuestro medio, mide la actitud positiva o negativa hacia el yo, aquí se considera los aspectos relacionados a la estima o el rechazo. Se trata por lo tanto de una autoevaluación positiva o negativa de uno mismo.

Finalmente señalaremos que se han utilizado otras escalas en ciertos estudios pero que no llegan más que a convertirse investigaciones particulares. Se trata del IA Inventario de Autoestima para niños elaborada por EOS, Perú. El otro instrumento fue una Evaluación Cualitativa de la Autoestima utilizada por Sánchez (2016).

Ante esta realidad antes descrita, que no menciona a un autor como Clemens (1999) que tiene una concepción de la autoestima desde el desarrollo y de la satisfacción de las necesidades y que 
postula en su propuesta planes de mejora, hemos decidido revalorar el modelo, darlo a conocer y utilizarlo para elaborar una Escala de Autoestima para adolescentes y adultos. La misma es presentada en los anexos en su versión original, faltando someterla a los análisis de validez y confiabilidad que nos permita contar con una escala de medición de la autoestima práctica, válida y útil para conocer sobre todo la autoestima de los adolescentes universitarios que es una población que nos preocupa y a la cual atendemos en nuestra labor de consejería en la universidad y que necesita instrumentos para conocerse y continuar desarrollándose y madurar como estudiantes y futuros profesionales.

\section{La autoestima desde el modelo de Harris Clemens: una propuesta de medición.}

Clemens (1999) parte de la propuesta que el niño para desarrollar su autoestima debe satisfacer ciertas necesidades básicas. Estas son la vinculación, la singularidad el poder y las pautas. La propuesta de Clemens si bien es conocida no ha sido muy difundida. Hemos encontrado algunos estudios que la utilizan para sus investigaciones pero son pocos. Entre ellos tenemos el trabajo de Pozo (1995) que realiza un estudio de observación con niños, utilizando la propuesta de Clemens.

Para Clemens, la autoestima tiene un efecto en todas las etapas de la vida de las personas. Una persona con poca autoestima tiene una minusvalía comparable a la de cualquier persona incapacitada físicamente; cuando se tiene poca autoestima se posee también muy escasa capacidad para tener éxito en el aprendizaje, en las relaciones humanas y en cualquier otro orden de la vida.

Clemens postula que la autoestima es sentirse a gusto con uno mismo. Es saberse importante para alguien que al mismo tiempo es importante para uno. Es sentirse "especial", aunque uno no sepa decir por qué. Es sentirse por encima de todo y saber cómo hacer las cosas que debía. Implica sentirse pleno de proyectos, sentir que avanza hacia sus metas y que las mismas están sustentadas en sus principios y valores. Es fácil observar a alguien con autoestima en base a lo que hace y cómo lo hace. Es un sentimiento que se demuestra siempre con hechos.

Es importante diferenciar las características de las personas con mucha autoestima de las que tienen poca autoestima, veamos este cuadro de las diferencias en la autoestima según la propuesta de Clemens (2006)

\section{Características de las personas según su autoestima}

\begin{tabular}{|l|l|}
\hline Con mucha autoestima & Con poca autoestima \\
\hline Se siente orgullosa de sus logros & Evita las situaciones que le provoquen ansiedad \\
\hline Se comporta con independencia & Desprecia sus dotes naturales \\
\hline Asume responsabilidades con facilidad & Siente que los demás no lo valoran \\
\hline Afronta nuevos retos con entusiasmo & Echa la culpa de su propia debilidad a los demás \\
\hline Se siente capaz de influir sobre otros & Se deja influir por otros con facilidad \\
\hline Muestra amplitud de emociones y sentimientos & Se pone a la defensiva y se frustra fácilmente \\
\hline Siente que puede solucionar los problemas & Se siente impotente \\
\hline Se preocupa por los sentimientos de los demás & Tiene estrechez de emociones y sentimientos \\
\hline
\end{tabular}


Otro aspecto importante de la autoestima es su influencia en las relaciones interpersonales en la medida que a cualquier persona le agrada mantener relaciones con quienes es grato o positivo mantener una relación, donde el otro siente que gana y crece estando con el otro. De lo contrario, una persona con poca autoestima puede comportarse a la defensiva, puede llegar incluso a ser agresivo o por otro lado poco comunicativo. Todo esto hace que las personas en vez de buscar su compañía y relación terminan en realidad alejándose de ellos. Estas personas en su necesidad de tener la aprobación y afecto de losdemás muchas veces deterioran sus relaciones al sentirse frustrados por que los otros no los consideran y pueden llegar a pensar y confirmar internamente sus pensamientos negativos de sí mismos, con frases como: "Es cierto, no tengo amigos", "Nadie me llama", "Realmente no me quieren", "No soy un ser valioso o importante"...entre otras frases que se pueden repetir mentalmente. Es por ello, que el desarrollo de la autoestima es clave para todas las personas e importante en todas las edades, se estructura en la niñez pero puede seguir desarrollándose en la adolescencia y adultez.

Un punto importante en la autoestima de los adultos y que estudia Clemens es la que se refiere a los padres con baja autoestima. Estos padres se proyectan a través de sus hijos, buscando que sus hijos logren lo que ellos no consiguieron. Entonces los hijos de estos padres viven inseguros entre vivir de acuerdo a las expectativas de sus padres o de sus propias necesidades. Además debemos señalar que los padres con baja autoestima tienen niveles altos de ansiedad, la cual influye negativamente en la comunicación. Estos padres provocan en sus hijos confusión e inseguridad. Por otro lado, estos padres no saben elogiar, si lo hacen es muy poco o en exceso y en forma general, lo cual no ayuda a los hijos a entender que conductas deben por ejemplo mantener y cuáles no.

Los padres con poca autoestima dan mensajes confusos y contradictorios a sus hijos y esto hace de ellos personas inseguros frente a los retos de la vida. Esto se puede apreciar en los comportamientos de los adolescentes que no se encuentran por ejemplos seguros de su vocación o de la profesión que están estudiando, esto por que sus padres no los apoyan del todo y porque muchas veces con sus palabras o hechos los pueden boicotear para que sus hijos no logren el éxito que ellos no consiguieron por ellos mismos. Este es uno de los motivos adicionales para trabajar con la autoestima en todas las edades, los adolescentes e incluso los adultos que pueden sin saberlo ocasionar daño a sus hijos si su autoestima no es adecuada o es poca.

\section{Aspectos de la autoestima según Clemens:}

Clemens señala que en definitiva son las experiencias vividas por los individuos en su infancia y el tipo de vínculo que establecen con sus cuidadores, es decir sus padres o sus sustitutos, quienes influyen en la personalidad y sobre todo en el sentimiento que se tiene sobre uno mismo. Es por ello, que las relaciones y los vínculos que tanto los padres como los educadores establecen con los niños y adolescentes deben ser de calidad y positivos para influir adecuadamente en la autoestima de los mismos.

Clemens propone que el desarrollo de la autoestima se da en la medida que se desarrollan cuatro componentes que responden a la satisfacción de las necesidades que todo ser humano tiene 
desde el nacimiento y en el transcurso de su desarrollo. Así el autor sostiene, que el carácter sano, la personalidad equilibrada, las buenas relaciones interpersonales, el desarrollo de objetivos claros y habilidades afectivas necesarias, se dan cuando los individuos tienen lo que necesitan. Clemens concluye que las dificultades emocionales o sociales que se producen en el desarrollo de toda persona siempre son producto de una carencia que se ha producido en las experiencias vividas en la infancia de todo ser humano.

Clemens sostiene que cuando se han satisfecho ciertas condiciones la persona gozará de autoestima, de lo contrario la falta o carencia se debe a no haberse dado alguno o varios de los componentes que a continuación describimos:

A. VINCULACIÓN: se produce por la satisfacción que tiene la persona al establecer vínculos que son importantes para él y que los otros reconocen como importantes.

Son características de una persona con vinculación:

$\checkmark$ Formar parte de algo.

$\checkmark$ Estar relacionado con otros.

$\checkmark$ Estar identificado con grupos concretos.

$\checkmark$ Tener un pasado y una herencia personales, lo cual amplía sus vinculaciones a otros lugares y épocas.

$\checkmark$ Poseer algo importante.

$\checkmark$ Pertenecer a alguien 0 a algo.

$\checkmark$ Sentirse apreciado por aquellas personas con las que él establece sus relaciones.

$\checkmark$ Ser importante para los demás

$\checkmark$ Poseer un cuerpo sano en el que puede confiar.

B. SINGULARIDAD: es el resultado del conocimiento y respeto que la persona siente por las cualidades 0 atributos que le hace especial o diferente. Además esto se ve apoyado por el respeto y aprobación de estas cualidades por los demás.

Son características de una persona con singularidad:

$\checkmark$ Respetarse.

$\checkmark$ Saber que él es alguien especial.

$\checkmark$ Sentir que sabe y puede hacer cosas que los demás no saben ni pueden.

$\checkmark$ Saber que los demás le creen especial.

$\checkmark$ Ser capaz de expresarse a su manera.

$\checkmark$ Usar la imaginación y dar rienda suelta a su creatividad

$\checkmark$ Disfrutar del hecho de ser diferente, aprendiendo al tiempo y a no incomodar a los demás.

C. PODER: es el sentimiento que tiene la persona de disponer de los recursos, oportunidades y capacidades de modificar su vida de manera significativa. 
Son características de una persona con poder:

$\checkmark$ Creer que puede hacer lo que ha planificado.

$\checkmark$ Saber que cuenta con los recursos materiales para hacer su trabajo.

$\checkmark$ Sentir que tiene a su cargo las cosas importantes de su vida.

$\checkmark$ Sentirse contento cuando termina algo que estaba bajo su responsabilidad.

$\checkmark$ Saber tomar decisiones y resolver problemas.

$\checkmark$ Saber controlar sus emociones frente a situaciones de estrés.

$\checkmark$ Utilizar las habilidades que aprendió.

D. PAUTAS: se refiere a la habilidad de la persona para tener como referencia de su sistema de valores a diferentes modelos, que pueden ser humanos, filosóficos o prácticos, sobre los cuales sustenta su comportamiento y su vida en general.

Son características de una persona con pautas:

$\checkmark$ Tener modelos que le sirven para guiar su vida.

$\checkmark$ Saber distinguir entre lo positivo y lo negativo.

$\checkmark$ Tener valores y creencias que le sirven de guía para su conducta.

$\checkmark$ Tener amplia experiencia para enfrentar situaciones nuevas.

$\checkmark$ Tener habilidades en el trabajo que le permitan lograr sus metas y valorarlas.

$\checkmark$ Dar sentido a todo lo que le ocurre en su vida.

$\checkmark$ Sentir que los demás aprecian los valores y principios que rigen su vida.

$\checkmark$ Saber cómo aprender.

$\checkmark$ Valorar el orden en todos los momentos de su vida.

\section{Una propuesta de medición}

Ante lo anteriormente descrito y partiendo de la importancia de la autoestima para todo individuo hemos planteado una propuesta de medición que se sustenta en el modelo teórico de Clemens que hemos presentado en los párrafos anteriores.

En colaboración con los estudiantes de la primera y segunda promoción de Psicología de la universidad, 2016 y 2017 nos propusimos esta meta que ahora presentamos a la comunidad universitaria, esto es la Escala de Autoestima 12 PsiUmch.

\section{La Escala de Autoestima 12 PsiUmch:}

La escala está compuesta de 40 ítems, cada uno de ellos ha sido elaborado desde la perspectiva teórica de Clemens. Hemos otorgado 10 ítems para cada uno de los componentes de la autoestima. Es decir diez ítems para vinculación, 10 para singularidad, 10 para poder y 10 para pautas. Los hemos distribuido en una escala tipo Lickert de 3 valores donde NUNCA tiene el valor de 0 puntos, A VECES vale 1 punto y SIEMPRE vale 2 puntos. Además hemos otorgado dirección negativa a 
algunos ítems que en posteriores estudios puede ser considerada como una escala de veracidad de la prueba. Hemos elaborado unas normas generales y específicas que nos permiten diagnosticar los niveles de autoestima y también los diagnósticos para cada componente de la autoestima.

No se ha realizado aún la aplicación de la prueba para encontrar su nivel de validez y confiabilidad, que será motivo de un próximo estudio dedicado específicamente a este propósito que permita darle la utilidad que deseamos a la Escala 12 Psi.

\section{Descripción de la Escala:}

A continuación describimos la escala de Autoestima 12 Psi, con sus distintos componentes y sus itemes correspondientes.

1. VINCULACIÓN: mide la satisfacción que tiene la persona al establecer vínculos que son importantes para él y que los otros reconocen como importantes

Tabla 1: Ítems del Componente I: Vinculación

\begin{tabular}{|c|c|}
\hline $\mathbf{N}^{\circ}$ ITEMES & ITEMES \\
\hline 5 & Siento que mi familia respeta mi estilo de vida. \\
\hline 8 & Frecuentemente busco la aprobación de los demás \\
\hline 17 & Me cuesta afrontar la frustración \\
\hline 28 & Ante un problema me pongo ansioso y tenso \\
\hline 31 & Siento que soy importante para mi familia. \\
\hline 32 & Es fácil expresar mis sentimientos ante los demás. \\
\hline 35 & Mis seres queridos se preocupan por mí. \\
\hline 36 & Me cuesta relacionarme con los demás \\
\hline 37 & Siento que no me toman en cuenta. \\
\hline 40 & Me incomoda hablar de temas personales \\
\hline
\end{tabular}

2. SINGULARIDAD: mide el conocimiento y respeto que la persona siente por las cualidades 0 atributos que le hace especial o diferente. Además esto se ve apoyado por el respeto y aprobación de estas cualidades por los demás.

Tabla 2: Ítems del Componente II: SINGULARIDAD

\begin{tabular}{|c|c|}
\hline $\mathbf{N}^{\circ}$ ITEMES & ITEMES \\
\hline 1 & Me gusta expresarme con mi propio estilo frente a los demás \\
\hline 2 & Soy creativo/a al realizar mis actividades \\
\hline 15 & Utilizo mis habilidades en diferentes situaciones \\
\hline 16 & Insisto en que las cosas sean a mi modo \\
\hline 19 & Tengo poca iniciativa \\
\hline
\end{tabular}




\begin{tabular}{|c|c|}
\hline 20 & Prefiero dirigir los grupos \\
\hline 26 & Me cuesta alcanzar los objetivos que me propongo \\
\hline 29 & Es difícil que los demás comprendan lo que quiero comunicar \\
\hline 38 & Es difícil entender a los demás. \\
\hline 39 & Evito participar en actividades grupales o numerosas \\
\hline
\end{tabular}

3. PODER: mide el sentimiento que tiene la persona de disponer de los recursos, oportunidades y capacidades de modificar su vida de manera significativa

Tabla 3: Ítems del Componente III: PODER

\begin{tabular}{|c|c|}
\hline $\mathbf{N}^{\circ}$ ITEMES & ITEMES \\
\hline 4 & Decido por mí mismo como organizar mis tareas \\
\hline 7 & Necesito que me digan con exactitud lo que debo de hacer \\
\hline 9 & Dejo que los demás decidan por mí \\
\hline 11 & Planifico y logro las metas que me propongo \\
\hline 12 & Me gusta asumir responsabilidades \\
\hline 13 & Tomo decisiones acertadas \\
\hline 14 & Me siento capaz de resolver problemas \\
\hline 18 & Evito aceptar responsabilidades \\
\hline 23 & Confío--en que puedo resolver mis problemas. \\
\hline 30 & Me cuesta tomar decisiones acertadas \\
\hline
\end{tabular}

4. PAUTAS: mide la habilidad de la persona para tener como referencia de su sistema de valores a diferentes modelos, que pueden ser humanos, filosóficos o prácticos, sobre los cuales sustenta comportamiento y su vida en general.

Tabla 4: Ítems del Componente IV: PAUTAS

\begin{tabular}{|c|c|}
\hline$N^{\circ}$ ITEMES & ITEMES \\
\hline 3 & Respeto la forma de ser de otras personas \\
\hline 6 & Me quedo en mi zona de confort por mis miedos \\
\hline 10 & Copio las ideas de las personas que influyen en la sociedad \\
\hline 21 & Soy capaz de darme cuenta entre lo que es bueno y lo que es malo \\
\hline 22 & Mi comportamiento esta guiado por valores y creencias. \\
\hline 24 & Me resulta difícil adaptarme a los cambios. \\
\hline 25 & Mis creencias son escuchadas y respetadas. \\
\hline 27 & Soy ordenado(a) en mis actividades cotidianas \\
\hline 33 & Valoro mis raíces familiares. \\
\hline 34 & Pienso que soy importante para la sociedad. \\
\hline
\end{tabular}




\section{Calificación de la Escala de Autoestima 12 Psi:}

Para la calificación de la Escala de Autoestima se otorgan los siguientes puntajes de acuerdo a la tabla 5 y se suman en su totalidad para la Escala general, es decir los cuarenta itemes. En el caso de los componentes, se suman los puntajes obtenidos en cada uno de ellos utilizando el puntaje de la tabla 5 y posteriormente se llevan a los criterios diagnósticos elaborados para la prueba original. No olvidar que las puntuaciones varían en los itemes que presentan dirección negativa.

Tabla 5: Puntajes de la Escala

\begin{tabular}{|c|c|}
\hline Escala & VALOR \\
\hline NUNCA & 0 \\
\hline A VECES & 1 \\
\hline SIEMPRE & 2 \\
\hline
\end{tabular}

\section{Criterios diagnósticos:}

Se ha elaborado dos criterios para la Escala Autoestima 12 Psi. El primero para la Autoestima Total, donde se suman todos los itemes de la escala y otra para los componentes de la autoestima donde se suman el grupo de diez itemes cada grupo. Veamos las criterios creados.

Escala Total: se presentan los puntajes posibles al contestar toda la prueba y tres criterios diagnósticos para la escala, tal como aparecen en la tabla 6.

Tabla 6: Criterios Diagnósticos - Escala Total

\begin{tabular}{|c|c|}
\hline PUNTAJES TOTALES & NIVELES DE AUTOESTIMA \\
\hline $70-80$ & ALTA \\
\hline $55-69$ & MEDIA \\
\hline $40-54$ & BAJA \\
\hline
\end{tabular}

Por componentes: se presentan los puntajes posibles al contestar cada uno de los componentes de la autoestima y los criterios diagnósticos para cada uno de ellos tal como aparecen en la tabla 7.

Tabla 6: Criterios Diagnósticos - COMPONENTES

\begin{tabular}{|c|c|}
\hline PUNTAJES COMPONENTES & NIVELES DEL COMPONENTE \\
\hline $15-20$ & ALTA \\
\hline $8-14$ & MEDIA \\
\hline $0-7$ & BAJA \\
\hline
\end{tabular}


En el anexo podemos apreciar la Escala de Autoestima 12 Psi, completa. Es una propuesta de medición de autoestima desde el modelo de Clemens. Es una escala a la que le falta realizar su validación y confiabilidad, pero es presentada a la comunidad científica para su revisión, mejora y completa los rigurosos análisis estadísticos que le permitan utilizarla. Ha sido creada con la intención fundamental de medir la autoestima en adolescentes a partir de 5to de secundaria, adolescentes universitarios y puede ser aplicada a adultos en general.

\section{Conclusiones:}

Se ha revalorado uno de los modelos teóricos que han estudiado la autoestima como uno de los factores más importantes en la personalidad de los seres humanos. Es decir el modelo teórico de Clemens que parte de la explicación de la importancia de la autoestima desde la perspectiva del desarrollo de cuatro componentes de la misma que se basan en las experiencias vividas por los individuos desde la primera infancia. Cuando uno de estos componentes no ha sido satisfecho, y se tiene poca vinculación, singularidad, pautas o poder vamos a observar las secuelas en la vida del individuo adolescente y adulto. Consecuencias en su desarrollo emocional o social, que pueden ser expresados en dificultades académicas, de relaciones interpersonales, laborales o incluso si son mayores en desajustes más serios, cercanos inclusive a problemas de salud mental.

Nuestra propuesta teórica y metodológica parte primero de explicar la importancia de la autoestima en nuestras vida y de elaborar un instrumento que al mismo tiempo que evalúa la autoestima y sus componentes permite crear de acuerdo a los resultados en cada uno de sus componentes, un plan o propuesta de mejora o desarrollo personal que permita al evaluado lograr sus objetivos y metas en la vida.

La propuesta, la Escala de Autoestima 12 Psi, en su versión original, que ahora presentamos, requiere ser sometida a los análisis estadísticos correspondientes, con la población objetivo de su elaboración. Se requiere aplicarla, analizar sus itemes, validarla con jueces, tal vez contrastarla con otras escalas de autoestima del medio. Es una tarea para completar en un próximo estudio, que nos motiva y compromete en la culminación del mismo.

Queremos concluir señalando que la autoestima puede y debe seguir siendo estudiada a partir de propuestas diferentes como la de Clemens, que le otorga tanta importancia a la historia y satisfacción de necesidades del individuo pero que al mismo tiempo le ofrece la oportunidad de mejorar su autoestima en base al desarrollo satisfactorio de estos componentes que son nucleares para entender todos los problemas de autoestima de los que somos testigos a diario en nuestra comunidad. 


\section{REFERENCIAS}

Branden, N. (1995). Cómo mejorar su autoestima. Barcelona: Paidós.

Branden, N. (1993). El poder de la autoestima. Cómo potenciar este importante recurso psicológico. Barcelona: Paidós.

Clark, A.; Clemes,H. y Bean, R.(2000). Cómo desarrollar la autoestima en los adolescentes. Madrid: Debate.

Clemens, H. \& Bean, R.(1995) Cómo desarrollar la autoestima en los niños. Madrid: Debate.

Clemens, H. \& Bean, R.(2000) Cómo desarrollar la autoestima en los Adolescentes. Madrid: Debate

Castañeda, A. (2013) Autoestima, claridad de autoconcepto y salud mental en adolescentes de lima metropolitana. (Tesis Licenciada en Psicología) PUCP. Lima.

Muñoz, L. (2011) Autoestima, factor clave en el éxito escolar: relación entre autoestima y variables personales vinculadas a la escuela en estudiantes de nivel socio-económico bajo. (Tesis de Magister en Psicología) Universidad de Chile. Santiago

Panduro, L.\& Ventura, Y. ( 2013) La Autoestima y su Relación con el Rendimiento Escolar de los Fstudiantes de Tercer Grado de Secundaria de la Institución Educativa José María Arguedas del Distrito de San Martín de Porres. (Tesis de Licenciado en Educación) Universidad Nacional de Educación Enrique Guzmán y Valle.

Robles, L. (2012) Relación entre clima social familiar y autoestima en estudiantes de secundaria de una institucion educativa del Callao. (Tesis de maestria) Universidad San Ignacio de Loyola. Lima.

Rodríguez, I. (2013) La autoestima y su relación con el aprendizaje del idioma Inglés en estudiantes del segundo grado de secundaria de la InstituciónEducativa N²0799, Huaral.( Tesis de licenciatura) Universidad Nacional de Educación Enrique Guzmán y Valle.

Rodríguez, C. \& Caño, A. (2012) Autoestima en la adolescencia: análisis y estrategias de intervención International Journal of Psychology and Psychological Therapy, 12, 3, 389-403 2012 Printed in Spain. All rights reserved. Facultad de Psicología, Universidad de Málaga, España. Recuperado de: http:// www.ijpsy.com/volumen12/num3/337/autoestima-en-laadolescencia-anlisis-ES.pdf

Sánchez, S. (2016) Autoestima y conductas autodestructivas en adolescentes. (Tesis de Licenciatura en Psicología Clínica) Universidad Rafael Landívar. Guatemala Quetzaltenango Recuperado de: http://recursosbiblio.url.edu.gt/tesiseortiz/2016/05/42/Sanchez-Sindy.pdf 